\title{
Grons $\begin{array}{ll}\text { Research Square } & \text { They should not be considered conclusive, used to inform clinical practice, } \\ \text { or referenced by the media as validated information. }\end{array}$
}

\section{PD-1/PD-L1-negative tracheal mucoepidermoid carcinoma: A case report and systematic review of the literature}

\section{Ying Ying Liu}

Huashan Hospital Fudan University

Jian Guo

Huashan Hospital Fudan University

Ji Chen

Huashan North Hospital affiliated to Fudan University

Hai Xia Li

Fudan University Huashan Hospital Department of Nephrology

\section{Zeng Tao Wang}

Huashan North Hospital affiliated to Fudan University

Jie Liu

The University of Sydney Faculty of Medicine and Health

\section{Yi Gong ( $\nabla$ gongyi1978@hotmail.com )}

Huashan North Hospital affiliated to Fudan University https://orcid.org/0000-0002-7504-3203

\section{Research Article}

Keywords: mucoepidermoid carcinoma (MEC), tracheal mass, airway management, anesthesia intubation method, PD-1/PD-L1

Posted Date: April 28th, 2021

DOI: https://doi.org/10.21203/rs.3.rs-429977/v1

License: (c) (1) This work is licensed under a Creative Commons Attribution 4.0 International License. Read Full License 


\section{Abstract}

Background: Tracheal mucoepidermoid carcinoma is a rare form of non-small cell lung carcinoma and is defined as a tumor characterized by a combination of squamous, mucus-secreting, and intermediate cell types. This carcinoma is usually located in the lobar or segmental bronchus. Currently, surgery is the preferred treatment for this disease, which includes pneumonectomy, lobectomy, and sleeve lobectomy.

Case presentation: A 50-year-old Chinese male presented with cough, shortness of breath and hemoptysis, and the effect of antibiotic therapy was not good. Subsequently, the airway occupied lesion was found by chest CT, and he was transferred to our hospital for surgical resection. Histologically, the tumor contained squamous epidermal cells, mucoepidermoid cells and intermediate cells. Immunohistochemistrically, the tumor cells were positive for p63, CK5/6, CK7 and Ki67. However, the tumor is generally negative for TTF-1 and neuroendocrine markers. The patient had no recurrence 15 months after the surgery.

Conclusions: We report a rare case of mucoepidermoid carcinoma in the distal trachea in which the surgery was difficult and could not be performed like a traditional pulmonary resection. We first provide a comprehensive description of airway management and anesthesia intubation. After surgery, we reviewed the literature and found that PD-1/PD-L1 detection had never been reported in tracheal mucoepidermoid carcinoma. Therefore, we studied the PD-1/PD-L1 pathway in this patient, and the results were negative, which may indicate that potential adjuvant therapy with immune checkpoint inhibitors (ICls) is not useful in this case.

\section{Background}

As a malignant neoplasm, the WHO named mucoepidermoid carcinoma (MEC) in 1990, and it was first described as a separate pathological entity by Stewart in $1945^{[1,2]}$. MEC accounts for $<10 \%$ of all tumors of the salivary glands and mainly occurs in the parotid glands and small salivary glands. MEC originating from the trachea and bronchus is very rare; it accounts for $0.1-0.2 \%$ of primary lung tumors and is more prevalent in females (approximately 1.5 times) than in males. The onset age of tracheal MEC is relatively young, with the median age at presentation being approximately 40 years old ${ }^{[1]}$. The clinical symptoms are mostly atypical and mainly manifested as cough, hemoptysis, pneumonia, wheezing, fever, chest pain and other symptoms and signs; MEC is easily misdiagnosed as endobronchial tuberculosis, bronchitis or asthma $^{[1,3]}$. Currently, surgery is the preferred treatment for this disease, but the choice of follow-up treatment is still uncertain. Immune checkpoint inhibitors against programmed death 1/programmed death ligand 1 (PD-1/PD-L1) have revolutionized the treatment of several solid tumors, and PD-L1 was reported to predict the response to immunotherapy ${ }^{[4,5]}{ }^{3}$, however, no studies have reported the expression of PD-1/PD-L1 in MECs. In this report, we describe the diagnostic, anesthetic, and surgical management of a MEC patient with negative PD-1/PD-L1 expression in the distal trachea.

\section{Case Presentation}




\section{Clinical History}

A 50-year-old Chinese male complained of cough, shortness of breath and hemoptysis for 10 days. The situation did not improve with antibiotic therapy at a local hospital. He underwent chest computed tomography (CT), showing a polypoid tumor just above the main carina, and was transferred to our hospital. The patient was a smoker of 30 years, with an average of 20 cigarettes a day. No family history of malignancy was found. Physical examination demonstrated obvious retractions of the suprasternal, supraclavicular and intercostal regions.

\section{Test results}

Tumor markers were all normal, lung function showed flow limitation, and flattening was noted in both the inspiratory and expiratory limbs of the flow-volume loop (Table 1 and Figure 1A). Chest-enhanced CT found tracheal obstruction by a lobulated heterogeneous enhancing soft tissue measuring $2.2 \mathrm{~cm}$, with suspected breakthrough of the airway wall, and no obvious enlarged lymph nodes in the mediastinum (Figure 2A-D). Flexible bronchoscopy (Figure 3A, 3B) was performed and revealed a mass protruding from the distal posterior tracheal wall $1 \mathrm{~cm}$ proximal to the carina. The tumor had a broad base, and its surface was not smooth and easily bled. A biopsy was performed at the local site of the lesion, and the bronchoscopy results indicated papillary tumor and mild cell dysplasia.

\section{Operation and Pathological Examination Results}

On December 19, 2019, the patient was referred for surgical management and underwent right posterolateral thoracotomy resection. Airway management was relatively difficult, and endotracheal intubation (via the mouth) was performed below the level of the lesion under general anesthesia. The trachea was incised at the lower edge of the tumor after careful separation, the endotracheal tube was withdrawn above the lesion and replaced by an auxiliary tube inserted into the distal left bronchus of the lesion to maintain adequate ventilation, the tumor segment trachea was resected along the upper and lower edges, and a frozen section verified that the tracheal margins were free of the tumor. End-to-end anastomosis was performed on the posterior wall of the trachea, the auxiliary tube was withdrawn, and the anastomosis on the anterior wall was completed without difficulty, as shown in Figures 4-5. Grossly, the tumor was an exophytic endobronchial circumscribed mass approximately $3 \mathrm{~cm}$ long and scaly with a hard white matter that blocked approximately $70 \%$ of the lumen. Histological staining showed squamous epidermal cells, mucoepidermoid cells and intermediate cells (Figure 6A-D). Immunohistochemical staining showed CK (+), P63 (+), HCK (+), ViM (-), LCA (-), CD56 (-), TTF-1 (-), P53 $(+)$, SY (-), NapsinA (-), S100 (-), Ki67 (5\%+), Calponin (-), CK5/6 (+), CK7 (small amount +), and PAS (-) 7-9: CK (-). The PD-1 and PD-L1 tests were negative (Figure 7). A diagnosis of primary tracheal MEC was finally determined, and no mediastinal lymph node involvement was observed.

\section{Post-Operative follow-up}


Postoperative recovery was satisfactory, and the outcome and surgical efficacy were investigated after 15 months of follow-up. Six months after surgery, activity endurance and pulmonary function (Table 1 and Figure 1B) were significantly improved, and no discomfort was reported. Chest CT (Figure 2E, 2F) and bronchoscopy (Figure $3 \mathrm{C}$ ) indicated that the trachea was unobstructed and that there was no tumor growth. The patient subsequently returned to a local hospital due to poorly differentiated MEC for radiotherapy from July to September 2020. At the last telephone follow-up in March 2021, the patient was well without any signs or symptoms of recurrence.

\section{Discussion And Conclusions}

The incidence of tracheal MEC is low. While still controversial, MEC is generally believed to originate from the ductal epithelium of the submucosal glands of the bronchus ${ }^{[6]}$. Because the tumor mostly occurs in the lobar or segmental bronchi and especially in the peripheral lungs ${ }^{[7]}$, the early stage is asymptomatic, and a small number of patients were noted only during routine health examinations. As the tumor grows and blocks the bronchial lumen, the patient presents with clinical symptoms of airway obstruction, such as cough, sputum coughing and progressive shortness of breath, which are easily misdiagnosed as endobronchial tuberculosis, bronchitis or asthma ${ }^{[1,3,8]}$. The patient we reported had obvious symptoms because the tumor was large enough and located in the distal trachea.

CT scans and bronchoscopy represent the main diagnostic tools for this disease, but the misdiagnosis rate of bronchoscopy biopsy is relatively high. Hsieh et al. reported that in a retrospective study of 41 cases of pulmonary MEC, only 4 patients had an accurate preoperative diagnosis from bronchoscopic biopsy ${ }^{[9]}$., which may be related to factors such as brittle tumors that bleed easily and small or shallow samples. The diagnostic gold standard for MEC is histopathological examination. There is no single immunohistochemical stain that one can define as pathognomonic for MEC ${ }^{[1]}$, but Roden $A C$ et al. reported that MAML2 rearrangement studies might be helpful in the distinction of MEC from other epithelial lung malignancies ${ }^{[10]}$. In this case, the tumor shows positive staining with antibodies commonly used for squamous cell carcinoma, such as p63 and CK5/6. In addition, the tumor may also show mild positive staining for CK7 and Ki67; however, the tumor is generally negative for TTF-1 and neuroendocrine markers. All of these findings are consistent with previous reports ${ }^{[1,11-13]}$.

A literature search was performed using the PubMed database to determine the availability of all full-text articles on tracheal MEC in English before April 2021. In total, 73 articles involving 83 cases were identified (Table 2); there were only 6 cases of tracheal lesions, accounting for $9.5 \%$, and the rest were all derived from bronchial lesions. Therefore, here we report the seventh case of trachea-derived MEC.

Treatment of tracheal MEC depends on its aggressiveness and the extent of its spread. Currently, surgical resection is considered the first treatment for this disease, which includes pneumonectomy, lobectomy, and sleeve lobectomy $[6,14]$. Of the 6 cases of tracheal MEC we retrieved, 2 cases were due to worsening upper airway obstruction, and emergency rigid bronchoscopy followed by electrocautery snaring was performed to remove the bulk of the tumor $[15,16]$. One patient injured the azygous vein and a branch 
from the right pulmonary artery leading to massive intratracheal bleeding, and an emergency sternotomy was performed. One patient received bronchoscopic polypectomy using a snare to relieve symptoms[17]. The other patient was a woman at 27 weeks of gestation, and she was treated by therapeutic bronchoscopy with argon plasma coagulation [18]. Only 2 patients underwent surgical resection, but no records of anesthesia or surgical procedures were reported $[19,20]$. In this report, we first provide a comprehensive description of airway management and anesthesia intubation because this case highlights the challenges with anesthesia and surgical interventions of large airways. Most cases have no recurrence in long-term clinical follow-up after complete tumor resection. Chemotherapy was used for patients with metastasis or inoperable evaluation. Some scholars also treat this disease with Epidermal Growth Factor Receptor (EGFR) inhibitors, suggesting that effective remission, recurrence delay and survival were achieved via EGFR inhibition $[3,21]$.

Important prognostic factors for MEC include histological type, TNM stage, radiation uptake and age[22]. Compared with high-grade MEC, low-grade MEC has a very good 5-year survival rate ${ }^{[23]}$. Fortunately, low grade MEC is dominant, accounting for $75.8 \%$ of the data in our statistics. Due to the rarity of tracheal MEC, there is no standardized approach to postoperative treatment. The majority of low-grade MEC cases were followed up regularly, while a few high-grade MEC cases were followed up with subsequent adjuvant chemotherapy or radiotherapy.

Immunotherapy, especially the use of immune checkpoint inhibitors (programmed death 1/programmed death ligand 1), has revolutionized the management of several different cancer types in recent years, and PD-L1 was reported to predict the response to immunotherapy ${ }^{[4,5]}$. In normal adult tissue, PD-L1 is constitutively expressed in normal placental trophoblasts as well as choriocarcinomas and trophoblastic components of germ cell tumors. Additionally, some neoplastic cells, such as squamous cell carcinoma of various sites, frequently express PD-L ${ }^{[24]}$. PD-L1 is only rarely expressed in adenocarcinoma cells and is not expressed in pulmonary schwannoma ${ }^{[24,25]}$. Normal lung parenchyma lacks PD-L1 expression, while lung carcinomas express varying levels of PD-L1 ${ }^{[24,26]}$; however, PD-1/PD-L1 expression has never been examined in tracheal MECs. Given the recent use of immune checkpoint inhibitors, as well as different levels of PD-1/PD-L1 expression in different organs, we investigated the PD-1/PD-L1 pathway in this patient. Surprisingly, this tumor was negative for PD-1/PD L1 immunoreactivity, which may indicate that potential adjuvant treatment with immune checkpoint inhibitors would not have been useful in this particular case. Of course, our sample data are too small, and checkpoint inhibitors remain to be tested in large clinical samples.

In summary, we present a case of PD-1/PD-L1-negative tracheal MEC, and describe anesthesia intubation and surgical procedures in detail. This kind of pulmonary malignant tumor is prone to misdiagnosis and missed diagnosis because of atypical clinical symptoms in the early stage. CT and bronchoscopy should be comprehensively applied to identify airway lesions, and patients should be treated as soon as possible. 


\section{Abbreviations}

\section{Abbreviations}

MEC mucoepidermoid carcinoma

CT computed tomography

PD-1/PD-L1 programmed death 1/programmed death ligand 1

EGFR Epidermal Growth Factor Receptor

\section{Declarations}

\section{Acknowledgements}

None.

\section{Ethics approval and consent to participate}

The study was approved by the ethics committee of the Huashan North Hospital Affiliated to Fudan University. The patient gave consent to participate.

\section{Consent for publication}

Informed consent was obtained from all individual participants included in the study.

\section{Availability of data and materials}

All the original data supporting our research are described in this article.

\section{Competing interests}

The authors declare that they have no competing interests.

\section{Funding}

Natural Science for Youth Foundation of China (Grant No. 81201835)

\section{Authors' contributions}

All authors contributed to the study conception and design. Yingying Liu and Jian Guo: data collection and manuscript writing. Ji Chen, Hai xia Li and Zeng tao Wang: result interpretation, literature search, and manuscript writing. Jie Liu: manuscript writing, proof reading. Yi Gong: mentoring of project, manuscript writing, and proof reading. All authors read and approved the final manuscript. 


\section{References}

[1] KALHOR N, MORAN C A. Pulmonary mucoepidermoid carcinoma: diagnosis and treatment. Expert Rev Respir Med.2018;12(3):249-255.

[2] YOUSEM S A, HOCHHOLZER L. Mucoepidermoid tumors of the lung. Cancer.1987;60(6):13461352.

[3] HOU J, WANG H, ZHANG G, et al. Mucoepidermoid Carcinoma of the Lung: Report of 29 Cases. Zhongguo Fei Ai Za Zhi.2017;20(3): 168-174.

[4] DUFFY M J, CROWN J. Biomarkers for Predicting Response to Immunotherapy with Immune Checkpoint Inhibitors in Cancer Patients. Clin Chem.2019;65(10):1228-1238.

[5] TIBALDI C, LUNGHI A, BALDINI E. Use of programmed cell death protein ligand 1 assay to predict the outcomes of non-small cell lung cancer patients treated with immune checkpoint inhibitors. World $J$ Clin Oncol.2017;8(4):320-328.

[6] FALK N, WEISSFERDT A, KALHOR N, et al. Primary Pulmonary Salivary Gland-type Tumors: A Review and Update. Adv Anat Pathol.2016;23(1):13-23.

[7] KUMAR V, SONI P, GARG M, et al. A Comparative Study of Primary Adenoid Cystic and Mucoepidermoid Carcinoma of Lung. Frontiers in Oncology.2018;8:153.

[8] ZHANG X P, HU P Z, SHEN S S, et al. Clinical characteristics and prognostic analyses of 87 patients with pulmonary mucoepidermoid carcinoma. Zhonghua Zhong Liu Za Zhi.2018;40(6): 452-455.

[9] HSIEH C C, SUN Y H, LIN S W, et al. Surgical outcomes of pulmonary mucoepidermoid carcinoma: A review of 41 cases. PLoS One.2017;12(5):e176918.

[10] RODEN A C, GARCIA J J, WEHRS R N, et al. Histopathologic, immunophenotypic and cytogenetic features of pulmonary mucoepidermoid carcinoma. Mod Pathol.2014;27(11):1479-1488.

[11] CHENG D L, HU Y X, HU P Q, et al. Clinicopathological and multisection CT features of primary pulmonary mucoepidermoid carcinoma. Clin Radiol.2017;72(7):610-611.

[12] SALEM A, BELL D, SEPESI B, et al. Clinicopathologic and genetic features of primary bronchopulmonary mucoepidermoid carcinoma: the MD Anderson Cancer Center experience and comprehensive review of the literature. Virchows Arch.2017;470(6):619-626.

[13] HUO Z, WU H, LI J, et al. Primary Pulmonary Mucoepidermoid Carcinoma: Histopathological and Moleculargenetic Studies of 26 Cases. PLoS One.2015;10(11):e143169.

[14] SHEN C, CHE G. Clinicopathological analysis of pulmonary mucoepidermoid carcinoma. World J Surg Oncol.2014;12:33. 
[15] HANIF K A, FAISAL M, MOHD A R, et al. Resolution of asthmatic symptoms following successful endoscopic resection of tracheal mucoepidermoid carcinoma. BMJ Case Rep.2019;12(1).

[16] SIGURDSSON M I, SIGURDSSON H, HREINSSON K, et al. Bronchiovenous fistula causing bleeding and air embolism: an unusual complication of bronchoscopic tumor resection. Am J Respir Crit Care Med.2011;183(5):681-682.

[17] SONOBE S, INOUE K, TACHIBANA S, et al. A case of pulmonary mucoepidermoid carcinoma responding to carboplatin and paclitaxel. Jpn J Clin Oncol.2014;44(5):493-496.

[18] KESROUANI A, DABAR G, RAHAL S, et al. Treatment of tracheal mucoepidermoid carcinoma by argon plasma coagulation during pregnancy. Int Surg.2015;100(5):927-929.

[19] MUSSI R K, TORO I F, PEREIRA M C. Mucoepidermoid carcinoma of the trachea mimicking asthma. J Bras Pneumol.2009;35(3):280-284.

[20] BREYER R H, DAINAUSKAS J R, JENSIK R J, et al. Mucoepidermoid carcinoma of the trachea and bronchus: the case for conservative resection. Ann Thorac Surg.1980;29(3):197-204.

[21] HAN S W, KIM H P, JEON Y K, et al. Mucoepidermoid carcinoma of lung: potential target of EGFRdirected treatment. Lung Cancer.2008;61(1):30-34.

[22] LI X, GUO Z, LIU J, et al. Clinicopathological characteristics and molecular analysis of primary pulmonary mucoepidermoid carcinoma: Case report and literature review. Thorac Cancer.2018;9(2):316323.

[23] KOMIYA T, PEREZ R P, YAMAMOTO S, et al. Primary lung mucoepidermoid carcinoma: analysis of prognostic factors using surveillance, epidemiology and end results program. Clin Respir J.2017;11(6):847-853.

[24] INAGUMA S, WANG Z, LASOTA J, et al. Comprehensive Immunohistochemical Study of Programmed Cell Death Ligand 1 (PD-L1): Analysis in 5536 Cases Revealed Consistent Expression in Trophoblastic Tumors. Am J Surg Pathol.2016;40(8):1133-1142.

[25] ZHOU D, XING X, FAN J, et al. PD-1/PD-L1 negative schwannoma mimicking obstructive bronchial malignancy: A case report. Thoracic Cancer.2020;11(8):2335-2338.

[26] CHEN Q, FU Y Y, YUE Q N, et al. Distribution of PD-L1 expression and its relationship with clinicopathological variables: an audit from 1071 cases of surgically resected non-small cell lung cancer. Int J Clin Exp Pathol.2019;12(3):774-786.

\section{Tables}

Table 1. Preoperative and postoperative lung function test. 


\begin{tabular}{|lllllll|}
\hline & \multicolumn{3}{c}{ preoperative } & \multicolumn{3}{l|}{ postoperative } \\
& Predict & Actual & Actual/Predict\% & Predict & Actual & Actual/Predict\% \\
\hline FVC (L) & 4.06 & 3.54 & 87.11 & 4.05 & 2.62 & 64.68 \\
\hline FEV1(L) & 3.32 & 1.27 & 38.33 & 3.31 & 2.52 & 76.21 \\
\hline FEV1/FVC (\%) & 81.78 & 35.93 & 43.93 & 81.73 & 96.13 & 117.62 \\
\hline mef25 (l/s) & 1.63 & 0.6 & 36.71 & 1.62 & 1.86 & 114.76 \\
\hline mef50 (l/s) & 4.24 & 0.85 & 20 & 4.23 & 3.47 & 82.15 \\
\hline mef75 (l/s) & 7.54 & 1.16 & 15.45 & 7.53 & 7.32 & 97.29 \\
\hline PEF (L/S) & 8.22 & 1.73 & 21.03 & 8.2 & 7.43 & 90.57 \\
\hline MVV(L/min) & 141.92 & 39.58 & 27.89 & 141.4 & 104.89 & 74.18 \\
\hline
\end{tabular}

Notes: FEV1, forced expiratory volume in one second; FVC, forced vital capacity; MEF75, MEF50, MEF25maximum expiratory flow rates were evaluated at the usual intervals of $75 \%, 50 \%$ and $25 \%$ of exhaled forced vital capacity; PEF-peak expiratory flow; MVV, maximum ventilatory volume.

Table 2. Summary of the clinicopathological features and treatment of patients. 


\begin{tabular}{|c|c|}
\hline Characteristics & $N(\%)$, median (range) \\
\hline \multicolumn{2}{|l|}{ Age (83 available) (years) } \\
\hline$\geq 18 \otimes 44$ available $\mathbb{Z}$ & $42.5 \rrbracket 20-81 \rrbracket$ \\
\hline$<18 \rrbracket 39$ available & $9 \llbracket 2-17 \rrbracket$ \\
\hline \multicolumn{2}{|l|}{ Sex (83 available) } \\
\hline Male & $53 \rrbracket 63.9 \rrbracket$ \\
\hline Female & $30 \rrbracket 36.1 \rrbracket$ \\
\hline \multicolumn{2}{|l|}{ Smoking history (29 available) } \\
\hline yes & $13 \rrbracket 44.8 \rrbracket$ \\
\hline No & $16 \rrbracket 55.2 \rrbracket$ \\
\hline \multicolumn{2}{|l|}{ Tumor location (63 available) } \\
\hline Trachea (above the carina) & $6 \otimes 9.5 \rrbracket$ \\
\hline main bronchi & $27 \rrbracket 42.9 \rrbracket$ \\
\hline Lobar bronchi & $21 \otimes 33.3 \rrbracket$ \\
\hline Segmental bronchi & $8 \otimes 12.7 \rrbracket$ \\
\hline Interlobar pleura & $1 \otimes 1.6 \rrbracket$ \\
\hline Tumor size $(\mathrm{cm})$ (46 available) & $3.19 \pm 1.77 \rrbracket 0.7-8.2 \rrbracket$ \\
\hline \multicolumn{2}{|l|}{ Pathology classification (62 available) } \\
\hline low-grade & $47 \rrbracket 75.8 \rrbracket$ \\
\hline high-grade & $11 \otimes 17.7 \rrbracket$ \\
\hline Intermediate-grade & $4 \rrbracket 6.5 \rrbracket$ \\
\hline \multicolumn{2}{|l|}{ treatment (80 available) } \\
\hline surgical resection & $66 \rrbracket 82.5 \rrbracket$ \\
\hline Therapeutic bronchoscopy & $8 \otimes 10.0 \rrbracket$ \\
\hline video-assisted thoracic surgery & $1 \rrbracket 1.25 \rrbracket$ \\
\hline Palliative chemotherapy and Radiotherapy & $5 \llbracket 6.25 \rrbracket$ \\
\hline \multicolumn{2}{|l|}{ Adjuvant chemotherapy (75 available) } \\
\hline Yes & $2 \varangle 2.7 \rrbracket$ \\
\hline No & 73凶97.3凶 \\
\hline
\end{tabular}

Page 10/15 


\begin{tabular}{|ll|} 
Outcome and follow-up (65 available) & \\
\hline CR & $56 \llbracket 86.2 \rrbracket$ \\
PR & $3 \rrbracket 4.6 \rrbracket$ \\
PD & $6 \rrbracket 9.2 \rrbracket$ \\
\hline
\end{tabular}

Notes: CR, complete response; PR, partial response; PD, progressive disease.

\section{Figures}

(A)

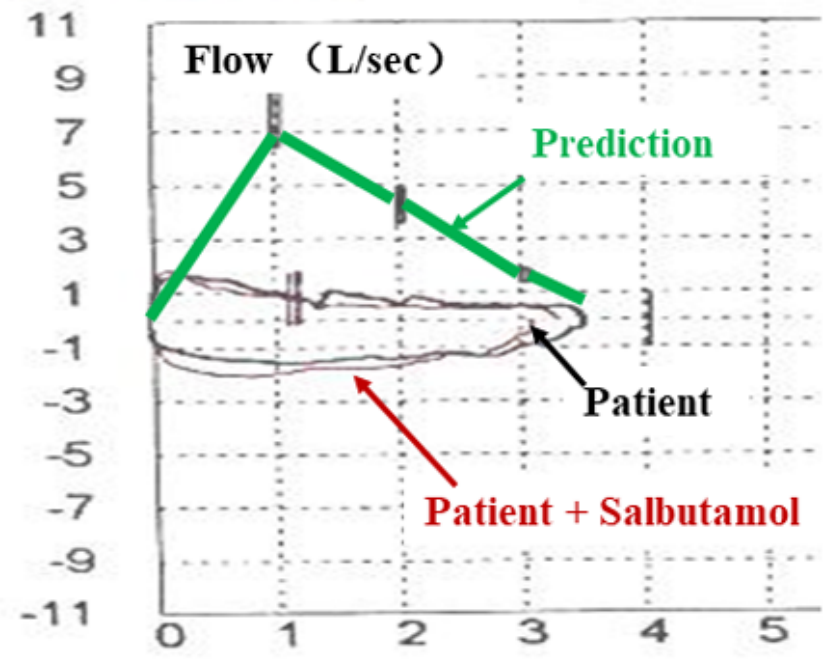

Volume (L)

(B)

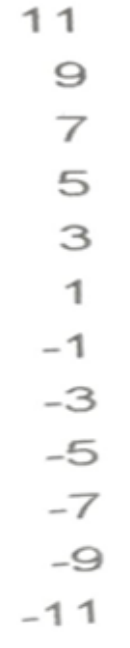

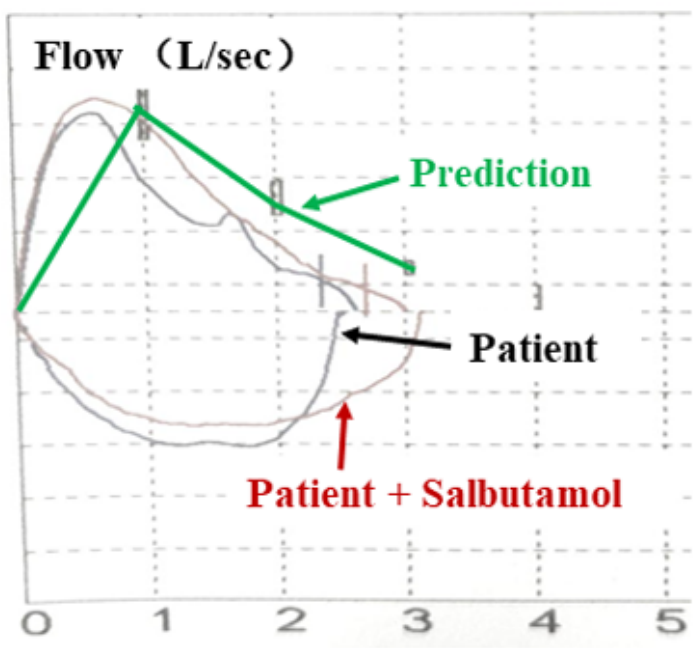

Volume (L)

\section{Figure 1}

Preoperative and postoperative lung function test. Notes: $(A)$ preoperatively, the F-V ring presents a characteristic platform shape, suggesting that inspiratory and expiratory flow is significantly limited; (B) postoperatively, pulmonary function indicates mild mixed ventilation dysfunction and normal small airway function. 
(A)

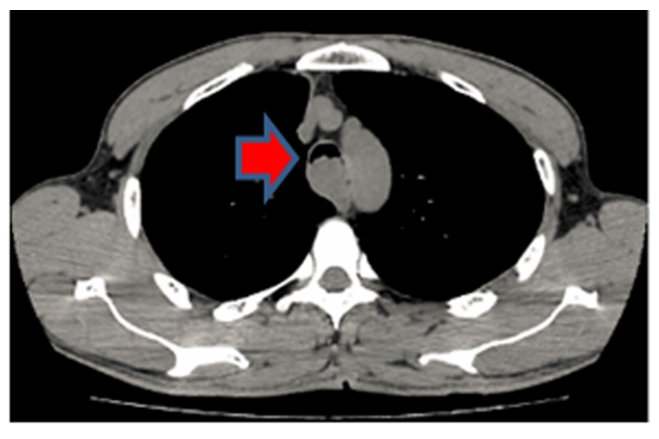

(B)

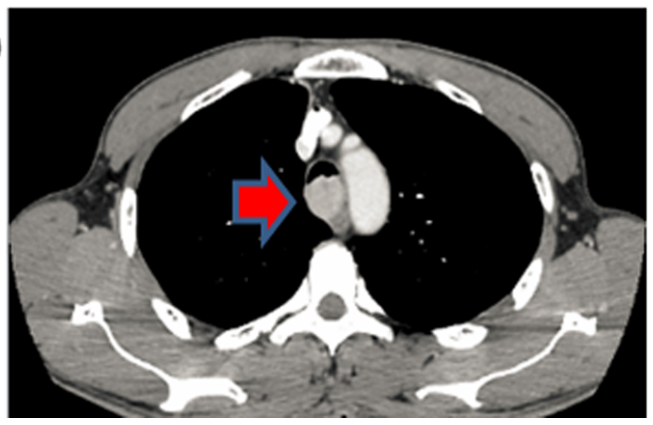

(C)



(D)

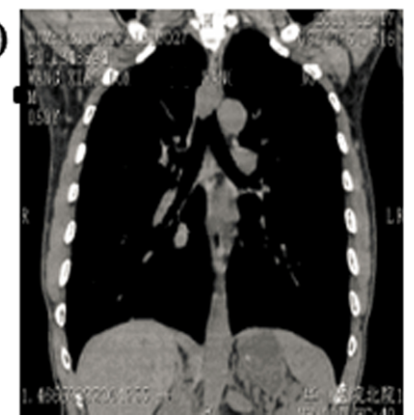

(E)

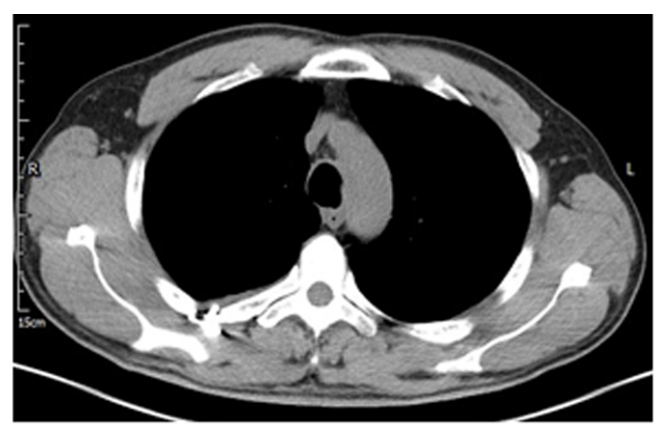

(F)



Figure 2

Chest CT demonstrating a polypoid endotracheal tumor. Notes: (A) (mediastinal window), (B) (enhanced $\mathrm{CT}$ ), (C) (coronary position, 3D reconstruction) and (D) (sagittal position, 3D reconstruction) preoperative chest CT images showing tracheal obstruction by a lobulated heterogeneous enhancing soft tissue lesion; (E) and (F) postoperative chest CT images showing that the tracheal was well without a tumor.

(A)

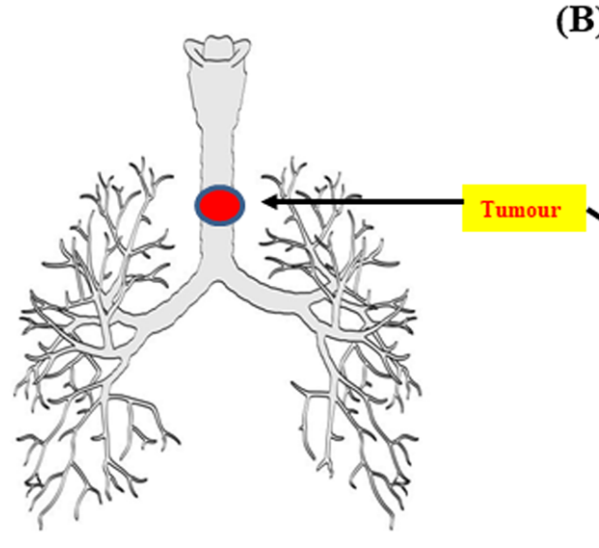

(B)

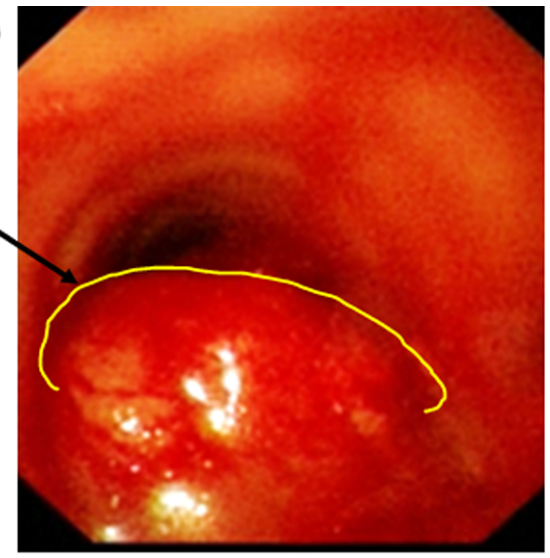

(C)

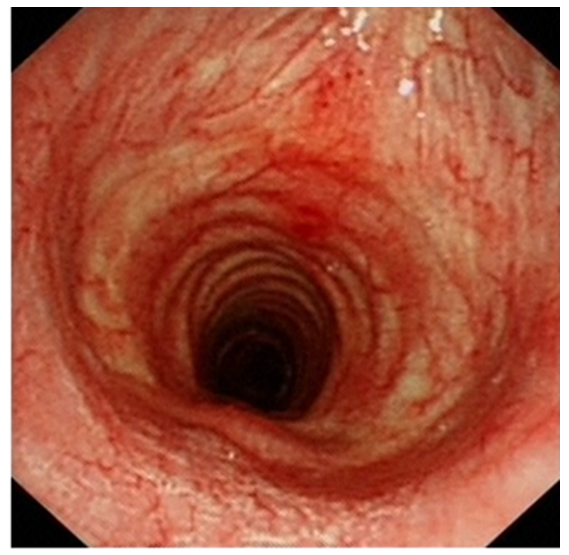

Figure 3

Bronchoscopic view of the tumor protruding into the trachea. Notes: (A) the tracheal tree pattern; (B) preoperative bronchoscopic image showing the $2.2 \mathrm{~cm}$ tracheal tumor that occluded the distal trachea; (C) postoperative bronchoscopy. 
(A) endotracheal intubation

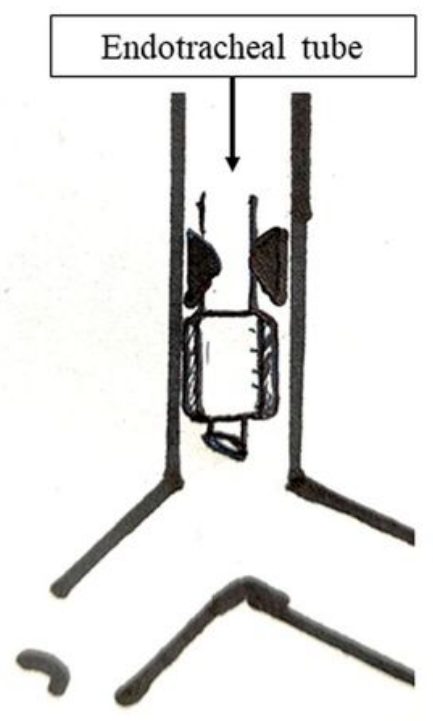

(B) incision \& auxiliary tube

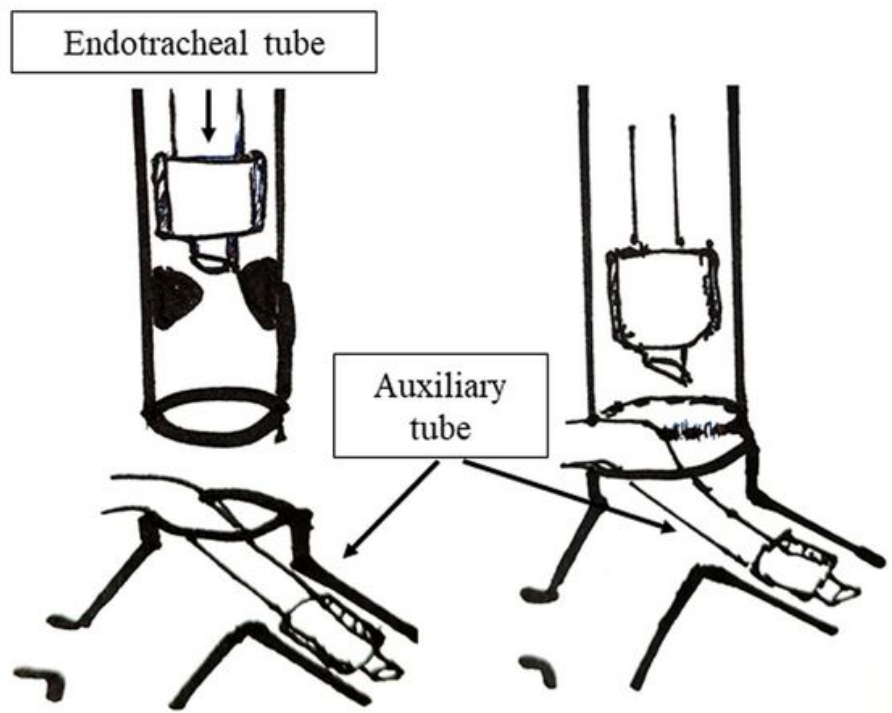

(D) closure

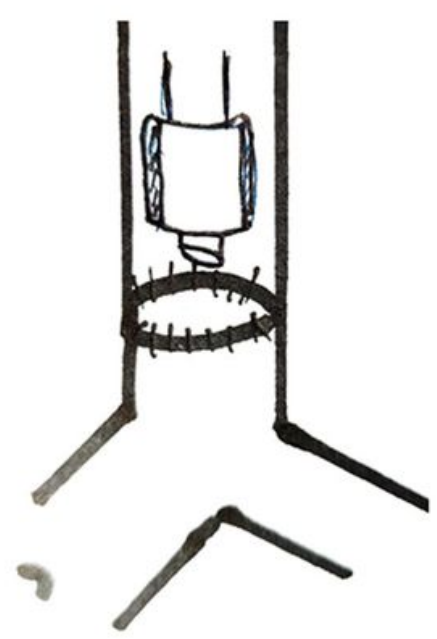

\section{Figure 4}

Anesthesia management, tracheal resection and reconstruction. Notes: $(A)$ endotracheal intubation (via mouth) was performed below the level of the lesion; (B) after tracheal resection, an auxiliary tube was inserted into the left bronchus distal to the lesion; (C) primary anastomosis; (D) after anastomosis, mechanical ventilation was maintained through an endotracheal tube. 
(A)

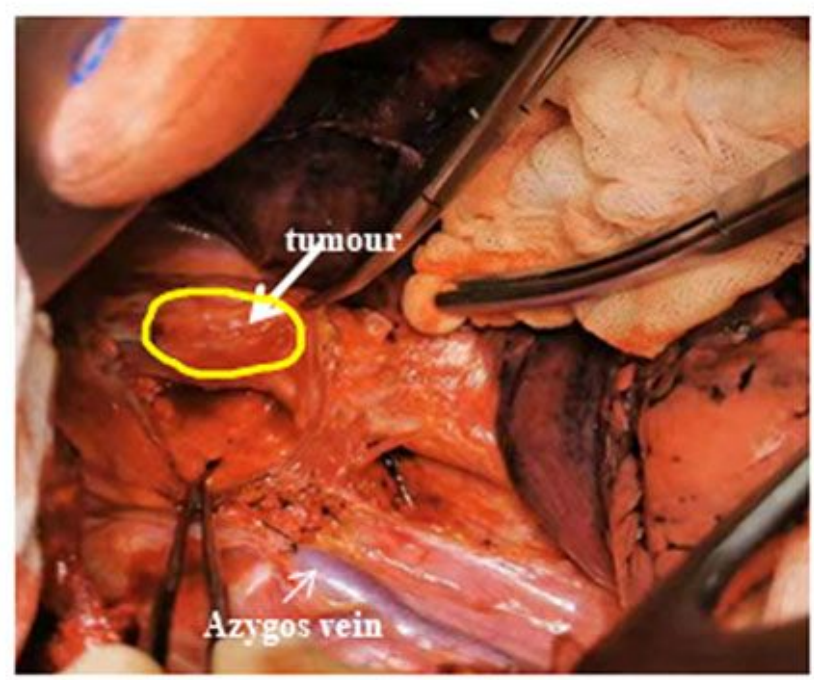

(C)

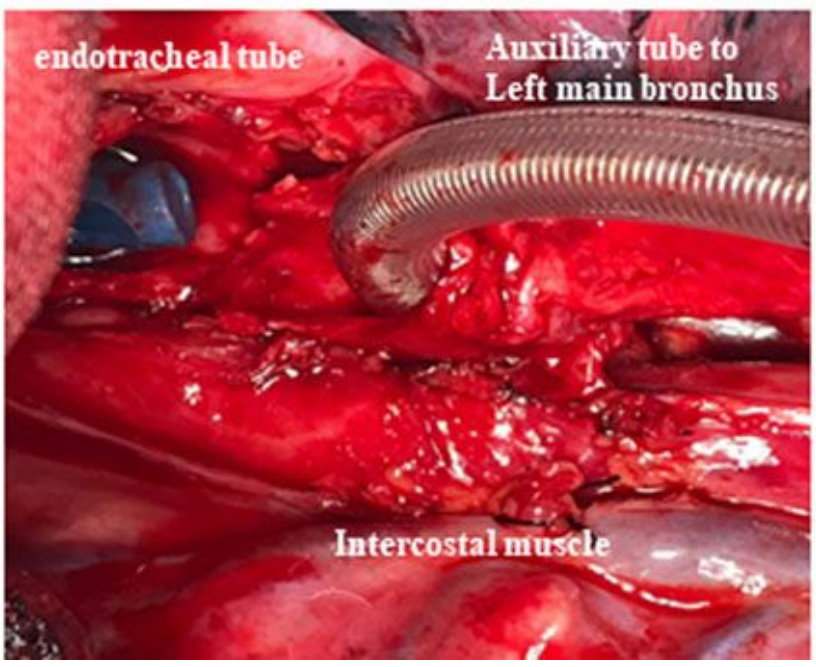

(B)

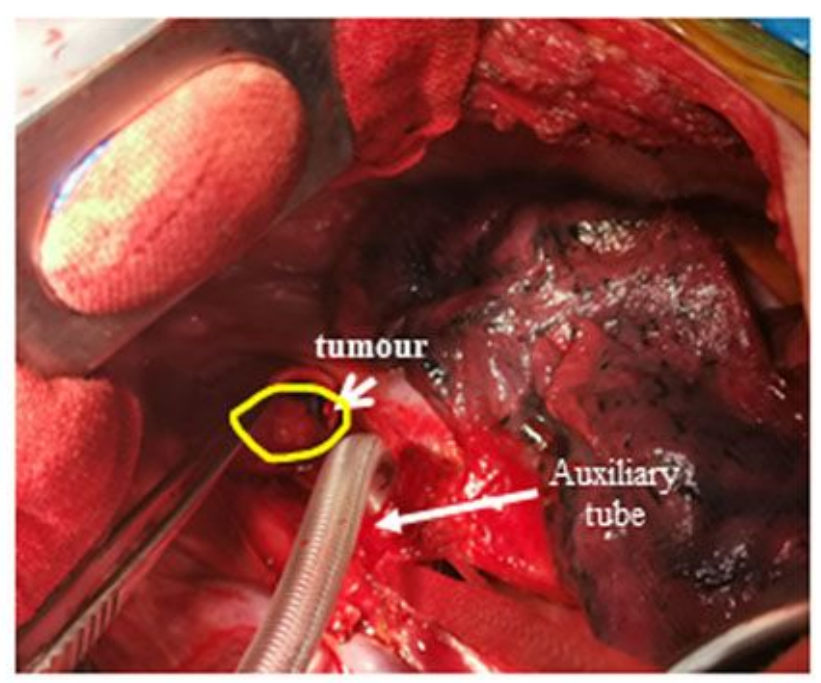

(D)

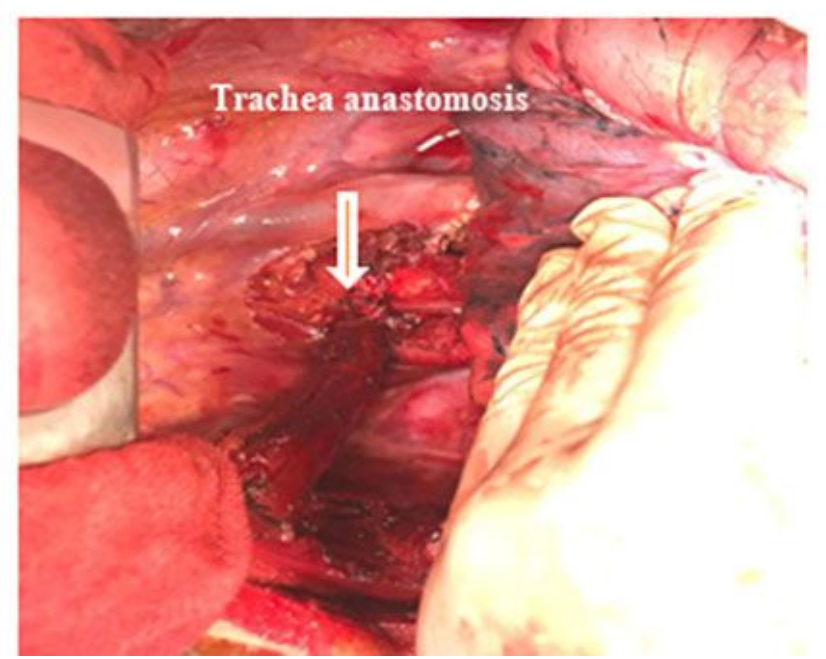

\section{Figure 5}

Tracheal resection and reconstruction. Notes: $(A)$ exposure of the trachea; $(B)$ intubation of the left bronchus with an auxiliary tube, the tumor is exposed in the trachea; (C) tumor resection and suture of the posterior wall of the broken end of the trachea; (D) free intercostal muscle and pedicel muscle flap to prevent anastomotic leakage. 

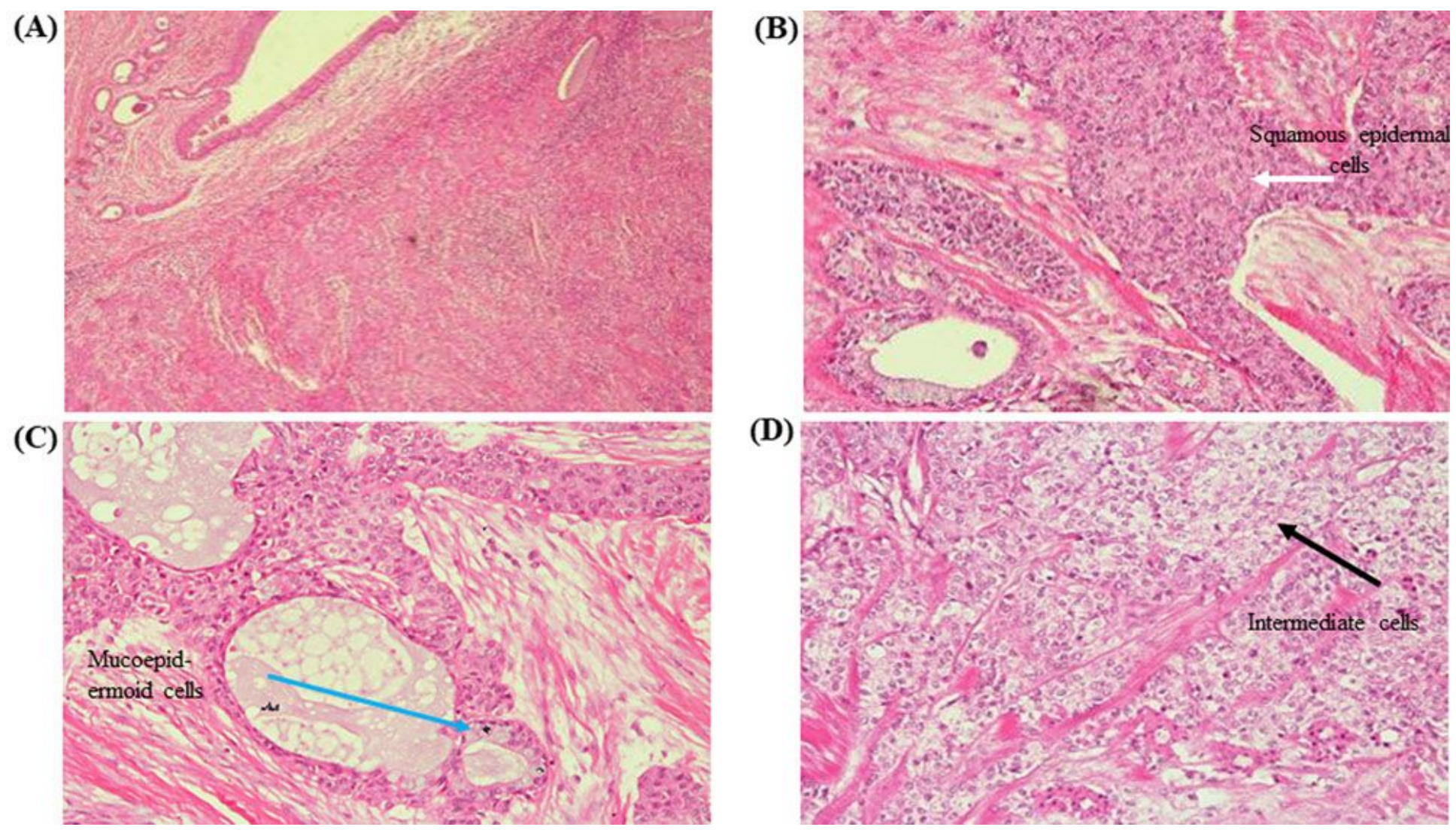

\section{Figure 6}

Pathology of the tracheal resection. Notes: (A) HE 40x mucoepidermoid carcinoma; (B) HE 100x; (C) HE 200x; (D) HE 200x; squamous epidermal cell (white arrow), mucoepidermoid cell (blue arrow), intermediate cell (black arrow).

(A)

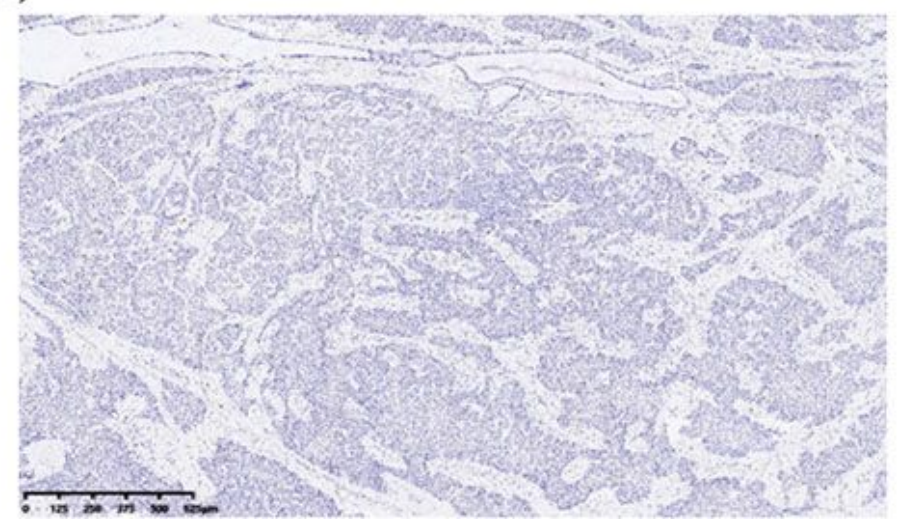

(B)

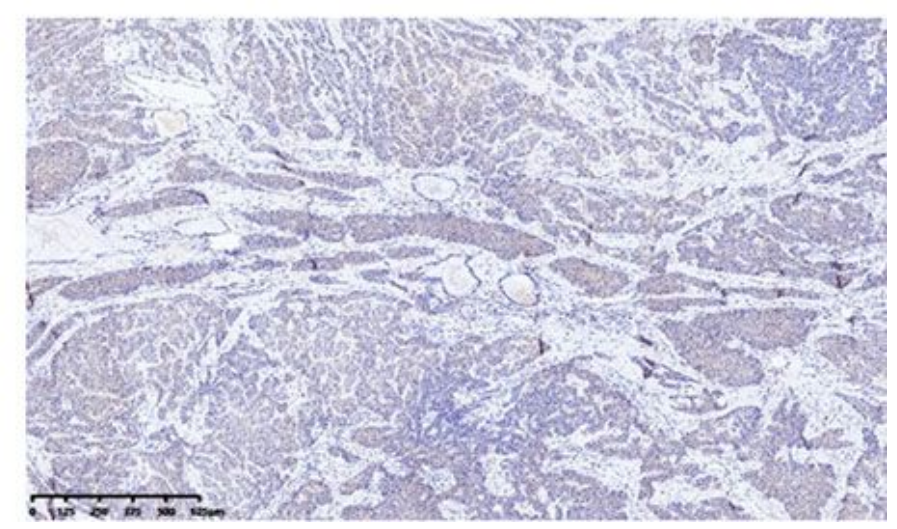

\section{Figure 7}

PD-1/PD-L1-negative tracheobronchial mucoepidermoid carcinoma. Notes: (A) PD-1 40x; (B) PD-L1 40x. 